\title{
Numerical Analysis of Thermal Management for High Power LED Array
}

\author{
Shen Yingdong ${ }^{1 *}$, Dai Junfeng ${ }^{1}$, Wang Yanlong ${ }^{1}$ \\ ${ }^{1}$ AVIC Chengdu Aircraft Industrial (Group) Co., Ltd., Chengdu 610092, China
}

\begin{abstract}
Adequate thermal management to remove and dissipate the heat produced by the LED is one of the main challenges in designing LED applications. In view of the above problems, this paper analyzed a heat sink as a heat exchanger for the LED array via the experiment combined with the numerical simulation. The results show that the heat sink is necessary for the LED array to guarantee reliable and safe operation. Moreover, the influence of the height of heat sink on the heat transfer of the LED array is also analyzed, and the optimized height of the heat sink for the 20W LED array is $20 \mathrm{~mm}$. Considering the heat transfer and the manufacturing cost, increasing the heat sink area blindly is not the best way to reduce the LED junction temperature, and more specific work should be considered.
\end{abstract}

\section{Introduction}

Light emitting diodes (LED), known as the fourth generation of illuminant, is one kind of electroluminescent semiconductor materials, which emits light by applying voltage exciting electrons ${ }^{[1]}$. Compared with traditional light sources, LED has higher energy efficiency, smaller size, longer lifetime and more environmental friendliness $[2,3]$. One of the main challenges in designing LED applications is ensuring adequate thermal management to remove and dissipate the heat produced by the LED and to guarantee reliable and safe operation. High temperature can damage the $p-n$ junction, lower the luminous efficiency, and shift the wavelength, which all negatively affect the lifetime of the LED. In contrast, low temperature is desirable because it severely influences the lifespan of LED, as an increase in junction temperature $\left(T_{j}\right)$ of $10 \sim 15$ ${ }^{\circ} \mathrm{C}$ can reduce the lifetime by $50 \%$.

As we all know, the luminescence of LED relies on electrons' transition between the energy band ${ }^{[4]}$. The heat produced by the LED cannot be dissipated in the form of radiation, but only in the form of heat conduction, and then, convection and radiation are conducted through the epitaxial equipment ${ }^{[5]}$. Especially, when the heat concentrated in a small chip, which cannot be spread out effectively, the temperature of the chip would rise rapidly, and thermal stress would not be distributed uniformly ${ }^{[6]}$.

Research shows that when the device temperature exceeds a certain value, the failure rate will rise exponentially ${ }^{[7]}$, since the increase $T_{j}$ would result in the light bias ${ }^{[8]}$, short life ${ }^{[9]}$, low luminous rate ${ }^{[10]}$. Most studies on the thermal management of LED have focused on the design of passive heat sinks to improve the thermal performance by reducing the spreading resistance. Ye et al. designed a heat sink with parallel vertical fins and three embedded heat pipes for high-power LED applications, which could limit the temperature of the heat sink base to about $70{ }^{\circ} \mathrm{C}^{[11]}$. Wang investigated the thermal characteristics of multichip LED modules under various chip distances. The experimental results showed that, under the same input power, the thermal resistance and junction temperature were higher in series than in parallel [12]. However, most of the analysis of the thermal management are only in the form of experiment, little involve simulations for their inner regularities.

In this work, we analyzes a high power array system for LED lights in the form of the simulations and the experiment, which used the varying height heat sink as a heat exchanger to dissipate heat to the surrounding air. And the experimental results are in good agreement with the simulation results. Furthermore, the performances of maximum junction temperature were investigated under same power but different heat sink.

\section{Experiment description numerical simulation}

For the experiment part, as shown in Figure 1, the experimental device was consisted of five parts, namely power, stabilizer, hand-held FLIR infrared temperature data acquisition, high power LED array and LED aluminum plate. The stabilizer is composed of a pressure regulating circuit, control circuit and servo motor etc. The

*Corresponding author: yingdongshen@163.com 
voltage stabilizer is to make the output voltage stable and ensure the normal operation of the LED. $220 \mathrm{~V}$ AC power was conducted to the rated DC voltage for LED through the regulator, and the current was delivered to the high power LED array. For the high power LED array, the LED array power was $25 \mathrm{~W}$, and the aluminum plate was $1 \mathrm{~mm}$ in thickness and $47.5 \mathrm{~mm}$ in radius. The real-time distribution of temperature on the LED array aluminum plate was measured via hand-held FLIR infrared temperature data acquisition.

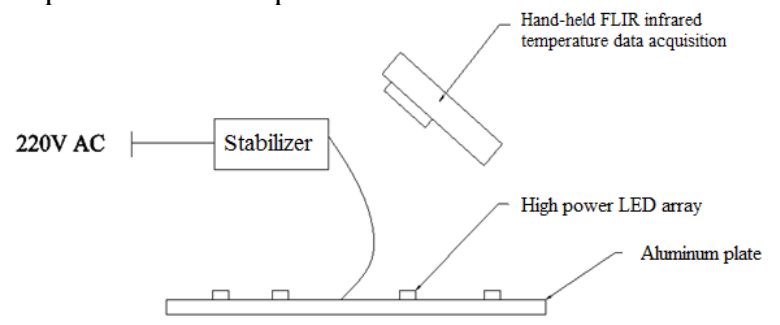

Figure 1. Experimental device

For the numerical simulation part, 25W LED array was taken, which was consistent with the experimental LED array. And following assumptions were made to simplify the calculation: LED is the uniform heat source, the top of LED is adiabatic, the initial temperature is $30^{\circ} \mathrm{C}$, and the experimental results show that air convection heat transfer is $12 \mathrm{~W} /\left(\mathrm{m} 2 \cdot{ }^{\circ} \mathrm{C}\right)$, and LED luminous efficiency is $20 \%$. In the process of numerical calculation, the physical model of LED is set up first, and the initial parameters of the model are set up. Then, according to the above hypothesis, the boundary conditions are applied to the physical model according to the Newton's law of cooling. Finally, the model is calculated by the finite element method.

\section{Results of the experiment and numerical simulation}

To measure distribution of temperature on the LED array aluminum plate (Figure 2), the light source was placed in a closed room to ensure the stability of the air convection coefficient, and the aluminum plate was maintained floating to ensure the natural convection effect in the aluminum substrate. When the lighting system reached the heat balance, the distribution of temperature on the LED array aluminum plate was recorded via hand-held FLIR infrared temperature data acquisition. During the whole process of the experiment, the experimental lamp was kept alone without any contact to ensure the stability of the lamp and air convection.

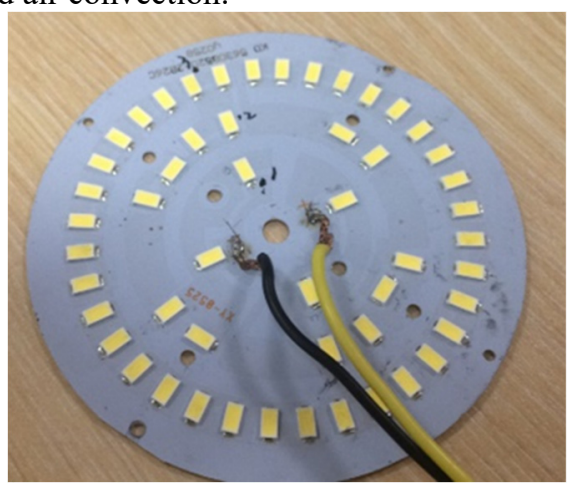

Figure 2. The photograph of the LED array.
To simulate the distribution of temperature on the LED array aluminum plate more accurately, three different measurement points was chosen and their temperature distributions were recorded. First, from the experiment results shown in Figure 3a), the temperatures of all measurement points on the LED array will increase when the LED lamps begin to work. After about $300 \mathrm{~s}$, the temperatures of all measurement points reach the peak $\left(\sim 95^{\circ} \mathrm{C}\right)$. Moreover, the temperatures of all points begin to fluctuate and LED lamps achieve heat balance in about $700 \mathrm{~s}$. In addition, the $T_{j}$ of point 1 is around $96.5^{\circ} \mathrm{C}$, point 2 is around $97.4^{\circ} \mathrm{C}$, and point 3 is around $97.5^{\circ} \mathrm{C}$.

Based on the above experimental results, numerical calculations are further performed. The numerical calculation was based on the following assumptions: LED is an uniform heat source, the top of LED is adiabatic, the initial temperature is $30{ }^{\circ} \mathrm{C}$, the air convection heat transfer is $12 \mathrm{~W} /\left(\mathrm{m} 2 \cdot{ }^{\circ} \mathrm{C}\right.$ ) (from the experimental results), and LED luminous efficiency is $20 \%$. As shown in Figure $3 \mathrm{~b})$, the temperatures of all the three points obtained from the numerical simulation match well with those from the experimental results, indicating that the numerical model used in the numerical calculation can simulate the $T_{j}$ of the LED array accurately during the operation of the LED array.
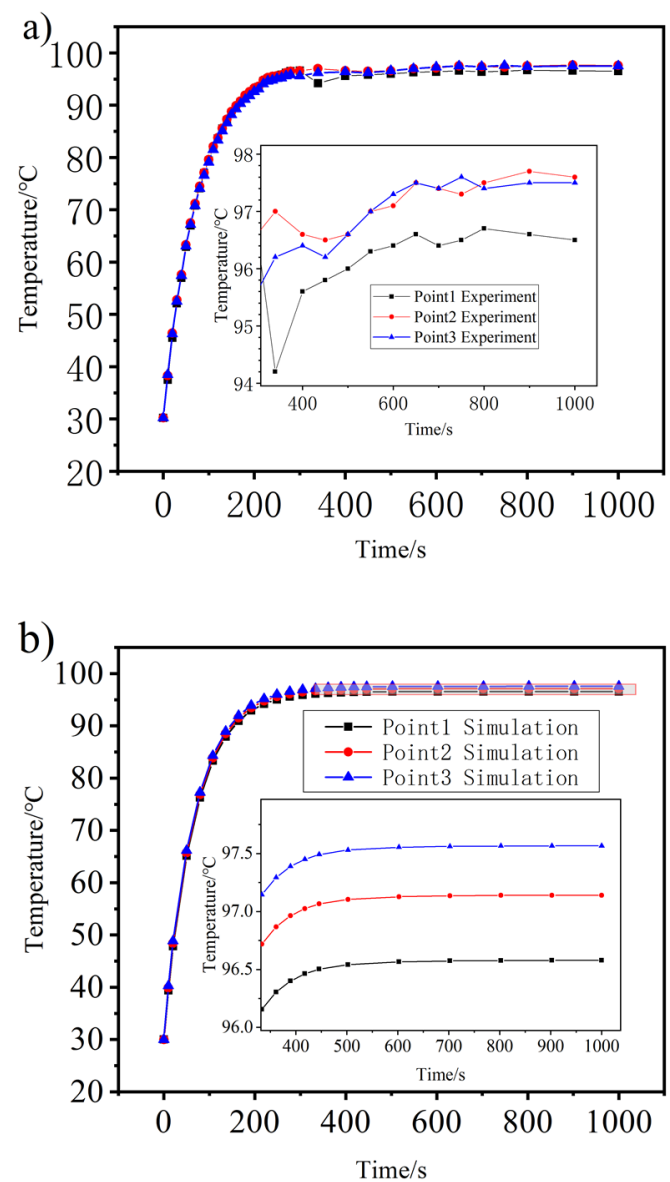

Figure 3. The distribution of temperature of three measurement part on the LED array via a) the experimental and b) the numerical simulation.

Furthermore, the distribution of temperature of the LED array at $1000 \mathrm{~s}$ is simulated. As shown in Figure 4, the temperature increases gradually from the inner part to 
the outer part with temperature range from $95.96{ }^{\circ} \mathrm{C}$ to $97.82^{\circ} \mathrm{C}$. Because of the optimum temperature for normal operation of the LED should be within $60{ }^{\circ} \mathrm{C}$, this high power LED array that without the heat sink auxiliary heat, seriously exceeds the normal operating temperature.

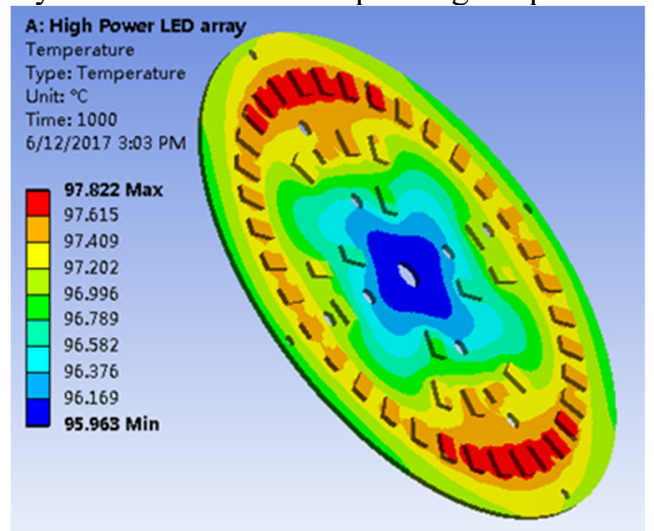

Figure 4. The distribution of temperature on the LED array via the numerical simulation.

\section{Optimization simulation}

via

numerical

To optimize the thermal management and ensure their reliable operation, $20 \mathrm{~mm}$ high heat sink is added to the original LED aluminum substrate. And the temperature of point 3 is simulated and recorded in Figure 5. By comparing the temperature changes at point 3 , the $T_{j}$ of high power LED array without heat sink is about $97.5^{\circ} \mathrm{C}$, while $T_{j}$ of high power LED array with heat sink reduces to $55^{\circ} \mathrm{C}$, namely the temperature of the LED array with heat sink decrease significantly $(\sim 57.89 \%)$. This decreased temperature is resulted from the increased heat dissipation area after adding the heat sink. All the above results indicate that adding the heat sink to the LED array is beneficial to reduce the $T_{j}$ and ensure their reliable operation.

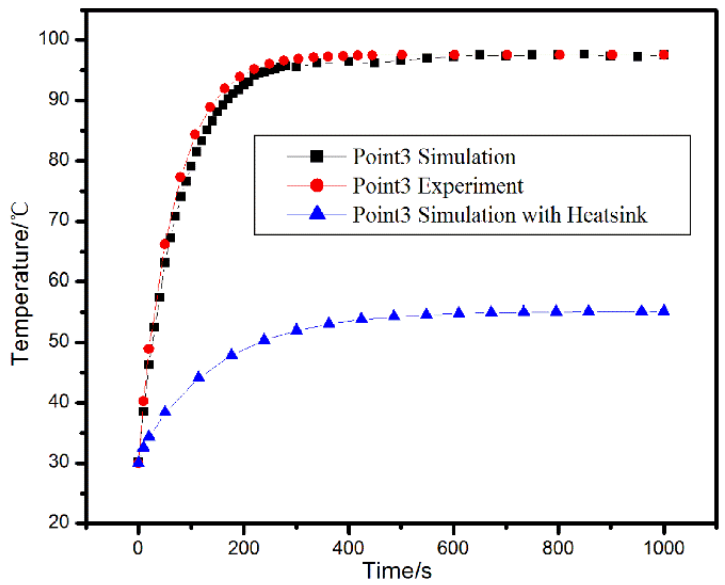

Figure 5. The temperature changes of point 3 on the LED array from the simulation result, experiment results and the temperature changes of point 3 on the LED array with heat sink from the simulation result.

The influence of the height of heat sink on heat transfer of the LED array is also examined. As shown in Figure 6, the LED array exhibits the less $T_{j}$ reduction at the same height reducing. And with the heat sink height increasing, the heat sink mass becomes larger, while increasing the cost of the array LED lamps also affect the overall structure. Therefore, in terms of heat sink, it is not the greater the better for the heat dissipation area, it should be combined with the actual work of the LED, and the most suitable heat sink should be selected according to the actual working temperature requirements.

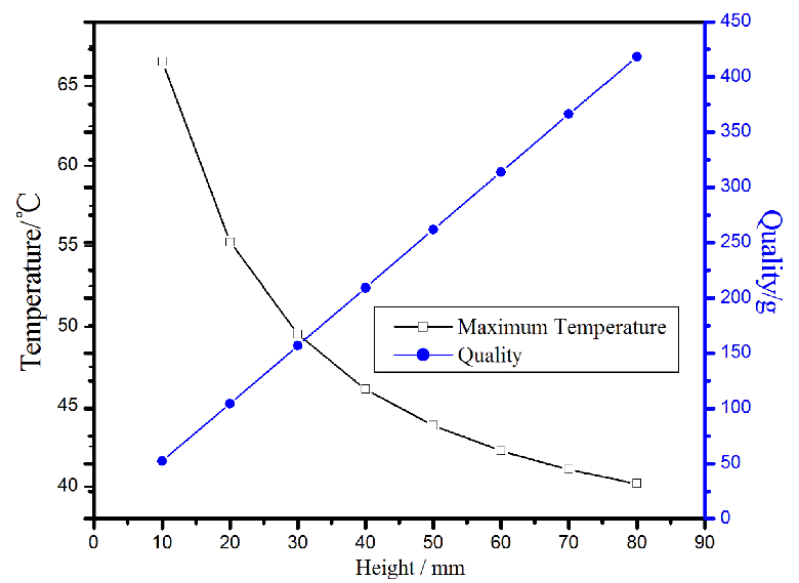

Figure 6. Influence of heat sink height on $T_{j}$ and mass of the LED array.

\section{Conclusion}

In this paper, we analyzed a heat sink as a heat exchanger for the LED array via the experiment combined with the numerical simulation. As for the LED array without the heat sink, the maximum $T_{j}$ reaches $97.5{ }^{\circ} \mathrm{C}$, which seriously exceed the LED normal working temperature of $60^{\circ} \mathrm{C}$. While after adding the heat sink with $20 \mathrm{~mm}$ height, the maximum $T_{j}$ of the LED array reduces to $55^{\circ} \mathrm{C}$. Therefore, it is necessary for LED to use the heat sink for auxiliary cooling. Moreover, the influence of the height of heat sink on the heat transfer of the LED array is also analyzed. The results indicate that a heat sink with $10 \mathrm{~mm}$ high reduces the maximum $T_{j}$ about $29^{\circ} \mathrm{C}$, while for that of $70 \mathrm{~mm}$, the $T_{j}$ is only reduced by $1{ }^{\circ} \mathrm{C}$, the LED array exhibits the less $T_{j}$ at the same height reduction. The heat gain of the heat sink is gradually reduced, while the manufacturing cost of the heat sink is gradually increased linearly. The $20 \mathrm{~W}$ LED array used in this paper has a perfect heat sink height at $20 \mathrm{~mm}$. Therefore, considering the heat transfer and the manufacturing cost, increasing the heat sink heat area blindly is not the best way to reduce the LED junction temperature, and therefore more specific works should be considered.

\section{References}

1. Xin Y. The Present Situation Analysis and Prosp ect of Enterprise Merger and Acquisition of Lig hting Appliance Industry in China [J]. China Illu minating Engineering Journal, 2017, 28(4)

2. Allan R. LED LIGHTING MOVES CLOSER T O THE MASS MARKET[J]. Electronic Design, 2011.

3. Liu M Q. The Comparison of Light Efficacy bet ween LED and Traditional Light Sources [J]. C 
HINA ILLUMINATING ENGINEERING JOUR NAL, 2006, 17(4).

4. Harbers G, Mueller G, Zhou L. Status and Futur e of High-Power Light-Emitting Diodes for Solid -State Lighting[J]. Journal of Display Technolog y, 2007, 3(2):160-175.

5. Shin H W, Lee H S. Analysis on thermal resist ance of LED module with various thermal vias [C].Physical and Failure Analysis of Integrated C ircuits. IEEE, 2011.

6. Christensen A, Graham S. Thermal effects in pa ckaging high power light emitting diode arrays [J]. Applied Thermal Engineering, 2009, 29(2-3): 364-371.

7. Yu B H. Junction Temperature and Thermal Res istance Restrict the Developing of High-power L ED [J]. CHINESE JOURNAL OF LUMINESCE NCE, 2005, 26(6)

8. Zhong W J, Wei A X. Dependence of GaN-bas ed White LED Colorimetric Parameters on Juncti on Temperature $[\mathrm{J}]$. Chinese Journal of Lumines cence, 2013, 34(9).

9. Narendran N,Gu Y.Life of LED-based white ligh $\mathrm{t}$ sources[J]. Journal of Display Technology, 200 5, 1(1):167-171.

10. Kim M H, Schubert M F, Dai Q, et al. Origin of efficiency droop in GaN-based light-emitting diodes[J]. Applied Physics Letters, 2007, 91(18): 15-67.

11. Ye H, Li B, Tang H. Design of vertical fin arra ys with heat pipes used for high-power light-emi tting diodes[J]. Microelectronics Reliability, 2014, 54(11):2448-2455.

12. Wang C P. Effects of Diode Voltage and Therm al Resistance on the Performance of Multichip L ED Modules[J]. IEEE Transactions on Electron Devices, 2016, 63(1):390-393. 